CHD incidence from the period 1980-1988 to 2000-2008 according to UK region. We also examine regional differences in changes in established coronary risk factors over these periods

Methods The British Regional Heart Study recruited 7735 men between 1978 and 1980 when aged 40-59 from 24 British towns. Established risk factors were measured at baseline examination and on 4252 participants twenty years later (1998-2000). CHD incidence over eight years from baseline was compared with incidence over eight years following re-examination. Age-adjusted Cox regression models including an interaction between an indicator for time period and region were used to assess relative hazards of CHD incidence between the two time periods according to UK region: South England (7 towns), Midlands/Wales (4 towns), North England (10 towns) and Scotland (3 towns).. Age-adjusted linear and logistic models with the same interaction term assessed changes in risk factors between the two periods according to region.

Results CHD incidence for 1980-1988 in the South, Midlands/ Wales, North and Scotland was 0.6, 0.8, 0.9 and 1.1 per 100 person years, and 1.1. 1.5, 1.3, and 1.1 for 2000-2008 when participants were 20 years older. Age-adjusted hazard ratios for the second versus first period in the four regions were $0.40,0.50,0.32$ and 0.22 ( $p$ for interaction $=0.05$ ), indicating that age-adjusted CHD incidence declined considerably in all regions but most rapidly among Scottish towns. While risk factor profiles were more favourable in the South than other regions in the first period, evidence of period/regional interactions were found in the analysis of some risk factors. Compared with the South, statistically significantly faster declines occurred in mean systolic blood pressure for North England and Scotland (by 3.1 $\mathrm{mmHg}$ and $4.1 \mathrm{mmHg}$ respectively), in mean total cholesterol for Midlands/Wales (0.24 mmol/l), in smoking for Scotland (ratio of odds ratios 0.74 ), and faster increases in moderate physical activity in North England and Scotland (ratio of odds ratios 1.20 and 1.41 respectively). However mean BMI increased similarly across all regions.

Conclusion Fall in CHD incidence was faster in the Scottish towns than the English towns. An impressive improvement in coronary risk profiles was likely to be responsible, especially with respect to blood pressure, smoking and physical activity.

\section{OP87 HEALTHY BEHAVIOURS IN MIDDLE AGE AND LONG- TERM CONSEQUENCES FOR MORTALITY, PHYSICAL AND COGNITIVE FUNCTION, AND MENTAL HEALTH}

doi:10.1136/jech-2012-201753.087

${ }^{1,2} \mathrm{I}$ A Lang, 'V Goodwin, ${ }^{3} \mathrm{R}$ Hubbard, ${ }^{4} \mathrm{DJ}$ Llewellyn. 'PenCLAHRC, Peninsula College of Medicine and Dentistry, Exeter, UK; ${ }^{2}$ Public Health Directorate, NHS Devon, Exeter, UK; ${ }^{3}$ Centre for Research in Geriatric Medicine, University of Queensland, Brisbane, UK; ${ }^{4}$ Epidemiology and Public Health Group, Peninsula College of Medicine and Dentistry, Exeter, UK

Background Physical activity, healthy body weight, smoking, and alcohol consumption are each related to mortality, physical and cognitive function, and mental health but their combined long-term effect is unknown. We examined the individual and combined influence of these risk factors on mortality and subjective and measured function in middle-aged adults followed for 18 years.

Methods Data were from the US Health and Retirement Study (HRS), a biennial, longitudinal, nationally representative survey of older adults. 11,597 participants aged 51 to 61 were followed from study enrolment for an 18 -year period. A health behaviour score was calculated with one point for each behaviour: moderate physical activity three times per week; BMI 20 to 25; alcohol consumption less than 7 drinks/week; non-smoking. Outcomes were all-cause mortality, problems with activities of daily living (ADLs), lung function, grip strength, walk speed, measured cognitive function, and depressive symptoms. Adjusted logistic and linear regression models were used to examine the relationships between health behaviours and outcomes separately by gender.
Results For all outcomes except grip strength there was a doseresponse relationship between healthy behaviour score and outcomes. For example, in men with four healthy behaviours and no baseline ADL problems, at 18-year follow-up $78.4 \%$ had no ADL problems, $6.6 \%$ had ADL problems, and $15.0 \%$ had died. In men with no healthy behaviours and no ADL problems at baseline, at follow-up $35.1 \%$ had no ADL problems, $8.8 \%$ had ADL problems, and $56.1 \%$ had died. Results in women were comparable but ADL differences were more marked. Number of healthy behaviours was related to follow-up cognitive function, mental health, walk speed, and lung function but not grip strength. Results were robust to adjustment for socioeconomic status (SES: measures of health, income, and level of education) and in analyses stratified by SES similar differences were found in relation to healthy behaviours within strata.

Conclusion Simple differences in lifestyle behaviours in middle age are associated with major differences in mortality, functioning and health risks as people progress into old age. Effective health promotion in these age groups could bring substantial health benefits for individuals across SES groups.

\section{OP88 THE HAZARD OF SMOKING FOR SPECIFIC CORONARY DISEASE PHENOTYPES: AN ELECTRONIC HEALTH RECORDS STUDY WITH LINKED DATA IN 915,000 PATIENTS}

doi:10.1136/jech-2012-201753.088

1J George, ${ }^{2}$ E Herrett, ' ${ }^{2}$ Denaxas, 'E Rapsomaniki, ${ }^{3} \mathrm{~A}$ Timmis, ${ }^{2} \mathrm{~L}$ Smeeth, ${ }^{1} \mathrm{H}$ Hemingway. ${ }^{'}$ Clinical Epidemiology Group, UCL, London, UK; ${ }^{2}$ Non-Communicable Disease Epidemiology Unit, London School of Hygiene \& Tropical Medicine, London, UK; ${ }^{3}$ NIHR Cardiovascular Biomedical Research Unit, Queen Mary University of London, London, UK

Background The association between smoking and coronary heart disease (CHD) has been well-documented, though most studies use a composite measure of CHD. Few studies have investigated the association between smoking and specific coronary disease phenotypes, even fewer, its first manifestation. These have generally been restricted to comparison between two disease phenotypes, often with small number of events or single gender studies. Our objective is to investigate the association between smoking and initial manifestation of specific coronary phenotypes, i.e. stable angina (SA), unstable angina (UA), non ST-elevation myocardial infarction (nSTEMI), ST-elevation myocardial infarction (STEMI), and coronary death unheralded by prior symptomatic disease (UCD), within a framework of competing risks for these and other atherosclerotic diseases.

Methods This was a prospective cohort study of $>915,000$ patients aged 30+ with no evidence of atherosclerotic disease in coronary, cerebrovascular or peripheral circulation prior to study entry. Using linked electronic health records from a CALIBER dataset, we incorporated primary care data from General Practice Research Data, acute coronary syndrome data from the Myocardial Ischaemia National Audit Project registry, hospital admissions data from Hospital Episode Statistics, and mortality and deprivation data from Office for National Statistics. The hazard of smoking was modelled using Cox proportional hazard regression, with data augmentation to incorporate competing risks of multiple disease presentations, and adjusted for age, sex, deprivation, blood pressure, blood-pressure lowering medication, diabetes and statin use.

Results There were 22,815 initial coronary disease presentations and a further 48,659 initial presentations of other cardiac, atherosclerotic cerebrovascular disease and peripheral arterial disease. Within the competing risk model, all coronary presentations showed an increased hazard for smokers compared to non-smokers, but the cause-specific adjusted hazard ratio (95\% CI) for ST elevation myocardial infarction (STEMI) $(2.99 ; 2.46-3.64)$ was substantially greater than those for the other presentations, including: stable angina: $(1.30 ; 1.25-1.35)$, unstable angina (1.48; 1.32-1.66), 